\title{
Advanced data analytics for building energy modeling and management
}

\author{
Cheng Fan' ${ }^{1}$, Fu Xiao² ( $\left.\square\right)$, Da Yan ${ }^{3}$ \\ (Guest Editors) \\ 1. Sino-Australia Joint Research Center in BIM and Smart Construction, College of Civil and Transportation Engineering, Shenzhen University, \\ Shenzhen, China \\ 2. Department of Building Services Engineering, The Hong Kong Polytechnic University, Hong Kong, China \\ 3. Building Energy Research Center, School of Architecture, Tsinghua University, Beijing, China
}

๑) Tsinghua University Press and Springer-Verlag GmbH Germany, part of Springer Nature 2020

The data-driven approach has been widely adopted in fault detection and diagnosis and optimal control of building energy systems for improving building performance since the 1980s, owing to the increasing demands for convenient and computationally efficient yet accurate models for online and automated applications. Conventional data-driven methods in the building field usually utilize a small amount of data (such as several hours or several days) which results in the applicability and generalizability of the data-driven models/knowledge being largely restricted by the scope of training data. Meanwhile, conventional data-driven methods still rely much on domain knowledge, such as selecting the model inputs and classifying data, which limits the capability of the data-driven approach in discovering new knowledge from data. The advance of the data-driven approach is restricted by data sources and the power of data analytics techniques.

Nowadays, buildings are not only energy intensive, but also information/data intensive. The wide adoption of information technologies, including both wired and wireless Internet and Internet of Things (IoT) in modern buildings has facilitated the collection and storage of a huge amount of operational data of building systems and equipment, as well as more environment and human behavior-related data. The effective use of the big building data becomes a challenge to and an opportunity for modern buildings facing the increasing complexity and integration of building and building energy systems, the increasing dynamic interactions among buildings, systems, occupants and power grids, and the increasing requirements of sustainability and intelligence.

\footnotetext{
E-mail: linda.xiao@polyu.edu.hk
}

This topical issue features the most recent work done by the active researchers in the building field on using advanced data analytics for building energy modeling and management. The authors adopted a diversity of advanced data mining and machine learning techniques, including neural networks, text mining, unsupervised clustering, fuzzy string matching algorithm, various regression techniques, support vector machine, boosting tree, random forest, kernel entropy component analysis, XGBboost algorithm, ensemble learning, reinforcement learning, transfer learning, etc., to discover novel data-driven knowledge from building data. The data sources include building automation systems, smart meters, social networks, public data sets and experiments. The volumes of data being analyzed obviously increased compared with previous studies adopting the data-driven approach. Two papers specifically looked at the usability and interpretability of the building data. Unsupervised clustering algorithms attracted substantial attention and are adopted in 5 out of 16 papers in this topical issue for grouping data into clusters with different energy or occupancy patterns. Unsupervised learning methods don't assign labels (such as input/output and normal/abnormal) to the original data and all the data are analyzed as a whole, which is beneficial to discovering previously unknown knowledge from the data. Text mining has been adopted by two papers which show the data type analyzed becomes diverse, not just numerical data. The data-driven knowledge obtained include building thermal behavior, energy patterns as well as occupancy patterns and behavior, which were used for providing more realistic occupancy-related inputs to building energy simulation, occupant-in-the-loop control, demand 
response as well as fault detection and diagnosis and optimal control of building systems and equipment, including variable air volume system, lighting system and chiller. The advanced data analytics are usually adopted to address big issues in buildings, like the whole building thermal response, interaction with smart grids and occupancy patterns, rather than the behavior of an individual component like heat exchangers, valves and fans in buildings. It is difficult even impossible to solve those issues only by physical knowledge as they involve complex dynamics and interactions. Datadriven knowledge is proved to be a valuable complement to domain knowledge in the building field.

This topical issue also includes a critical review paper on using the advanced data analytics from the data sources, data pre-processing, data mining and machine learning based knowledge discovery, knowledge interpretation and knowledge applications. The review paper provides a useful overall picture about the current status of $\mathrm{R} \& \mathrm{D}$ in the topic and the perspectives of big data and artificial intelligence in the field of building science and engineering.

Although advanced big data analytics are constantly emerging, their potentials in analyzing high-complexity and low-quality big building data have not yet been fully exploited. Big data and artificial intelligence technologies are driving innovations in modern buildings, which will bring powerful innovative tools for data analysis, modeling, control, optimization and diagnosis for smart buildings to deal with increasingly complex integration and interactions among buildings, systems, environment, occupants and smart grids. It is hoped that this topical issue will inspire more innovative research on using advanced data analytics for building energy modeling and management. 